\title{
PHOTOELECTRIC PROPERTIES OF HETEROSTRUCTURES BASED ON PEPC AND MEH-PPV FILMS DOPED WITH ZINC OCTABUTYLPHTHALOCYANINE
}

\author{
Nicolay Davidenko a, Irina Davidenko a*, Oleg Korotchenkov ${ }^{\mathrm{a}}$, Victor Kravchenko ${ }^{\mathrm{b}}$, \\ Elena Mokrinskaya ${ }^{a}$, Andrey Podolian ${ }^{a}$, Sergey Studzinsky ${ }^{a}$, Larisa Tonkopiyeva ${ }^{a}$ \\ ${ }^{a}$ Kiev Taras Shevchenko National University, 64, Volodymyrska str., Kiev 01601, Ukraine \\ ${ }^{b}$ Institute of Physical Organic Chemistry and Coal Chemistry, 50, Kharkivske Road, Kiev 02160, Ukraine \\ "e-mail: irynadavydenko@gmail.com; phone: (+38) 0674095537
}

\begin{abstract}
Planar organic heterostructures have been prepared using poly-N-epoxypropylcarbazole films and poly[2-methoxy-5-(2'-ethylhexyloxy)-1,4-phenylenevinylene] by the method of successive deposition adding $2,3,9,10,16,17,23,24$-zinc octabutylphthalocyanine. Photoelectric, photodielectric and photovoltaic properties of the heterostructures have been studied. It has been found that the structure photosensitivity in the red part of spectrum is remarkably enhanced by adding the metal complex into the $\pi$-conjugate polymer. It has also been established that metal sensitizers are capable of slowing down the photovoltaic rise and decay transients exhibited after the illuminating light is switched on and off, respectively. The likely origin of these effects is suggested, relating to deep traps generated by the metal complex.
\end{abstract}

Keywords: organic heterostructures, MEH-PPV, metal complexes, photovoltaic effect.

Received: February 2016/ Revised final: March 2016/ Accepted: March 2016

\section{Introduction}

Over the last decades, the considerable effort has been devoted to the development of low-cost and flexible organic photonic devices based on molecular composition of aromatic and heteroaromatic compounds absorbing light within the solar range. Quite recently, the superior photovoltaic performance has been demonstrated employing phthalocyanines, and the new class of conjugate polymers containing para-phenylenevinylene (PPV), porphyrin and fullerene fragments have been reported [1-4]. One of the most popular material of this type is poly[2-methoxy-5-(2'-ethylhexyloxy)-1,4phenylenevinylene] (MEH-PPV) with the long-wavelength absorption edge occurred at about $600 \mathrm{~nm}$.

It has been previously reported that MEH-PPV-based film structures reveal the photoelectric effect, and the photovoltage (PV) have been observed in these structures [5]. In order to shift the photosensitivity spectrum of this material to the near infrared, the polymer composite films (PCF) doped by organic dyes or metal complexes have been usually employed [6-11]. The occurrence of the light absorbing centres and the resulting generation of electron-hole $(e-h)$ pairs provide the pathways for the inner photoeffect sensitizing observed in PCF. The effectiveness of the lightelectricity transformation is mainly determined by the $e-h$ pair lifetime. The lifetime can be considerably enhanced by introducing the hetero-interface, consisting of two PCF with different electronic properties. In this case, the transport probability for carriers, moving from one PCF to the other one, depends upon the energy barriers occurring at the interface and can reduce the geminate recombination probability of the carriers.

This type of heterostructures can be designed using, e.g. MEH-PPV and carbazolyl-containing polymers or oligomers (for example, poly-N-epoxypropyl-carbazole, PEPC). The first structure possesses both the electron and whole conductivity, whereas the second one is solely of $p$-type. Despite the large number of papers regarding the photovoltaic devices with carbazolyl groups [12], there is a lack of experimental studies aimed to investigate the photoelectric properties of film heterostructures containing MEH-PPV and PEPC with sensitizing dopants.

Therefore, the purpose of this work was to develop the photosensitive PCF heterostructures based on PEPC and MEH-PPV doped with sensitizing 2,3,9,10,16,17,23,24-zinc octabutylphthalocyanine ( $\left.\mathrm{Pc}^{\mathrm{Bu}} \mathrm{Zn}\right)$. The photoconductive, photovoltaic and photo-dielectric properties of these devices have been investigated together with the photoexcited carrier dynamics.

\section{Experimental}

The starting materials were PEPC, MEH-PPV and $\mathrm{Pc}^{\mathrm{Bu} Z n}$ with the structural formulas shown in Figure 1. The glass substrates $\left(3 \times 2 \mathrm{~cm}^{2}\right)$ were initially cleaned with a liquid mixture of acetone and rinsed with distilled water. A thin $(\approx 1000 \mathrm{~nm})$ conductive Indium Tin Oxide (ITO, $\mathrm{SnO}_{2}: \mathrm{In}_{2} \mathrm{O}_{3}$ ) layer was deposited onto the substrate. The PEPC or MEH-PPV polymer films, with (concentration of $3 \mathrm{wt} \%$ ) or without metal additives, were then spin-coated from polymer solutions onto the ITO covered substrates and dried on air at $80^{\circ} \mathrm{C}$ during 24 hours. The thickness of polymer layers was $L=2.0 \mu \mathrm{m}$ in all the sample structures, as measured by an interference microscope. The structures were finally Ag-covered by a thermal evaporation technique. 

were used.<smiles>CCC(C)OCCCCO</smiles>

a)

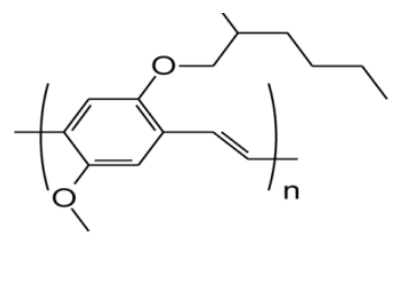

b)

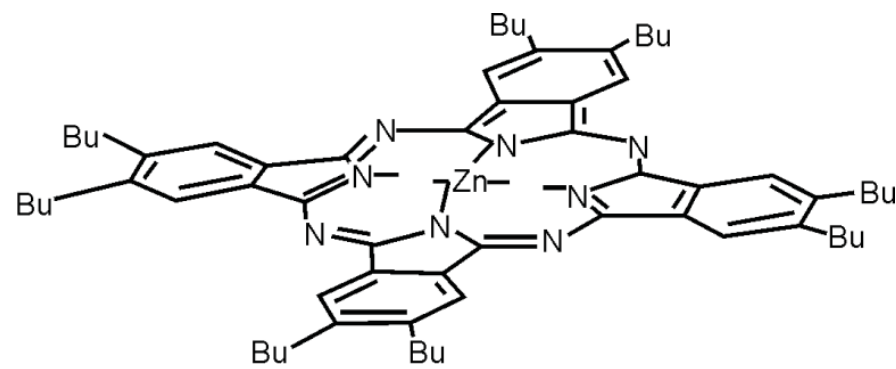

c)

Figure 1. Structural formulas of PEPC (a), MEH-PPV (b), $\mathrm{Pc}^{\mathrm{Bu}} \mathrm{Zn}$ (c).

Application of voltage $U(1$ to $300 \mathrm{~V}$ ) between the ITO and Ag electrodes (Figure 2) allows the current generation with the density of $j$ to flow in PCF. It changes when the layers are illuminated through the bottom side of the structures shown in Figure 2. Importantly, PEPC is colourless and it does not absorb in the visible part of the spectrum because its absorption falls within the near ultraviolet part of the spectrum. In turn, MEH-PPV absorbs effectively the light within $300-600 \mathrm{~nm}$ limits with a peak at about $500 \mathrm{~nm}$ (Figure 3, spectrum 1), whereas $\mathrm{Pc}^{\mathrm{Bu}} \mathrm{Zn}$ absorbs within the range from

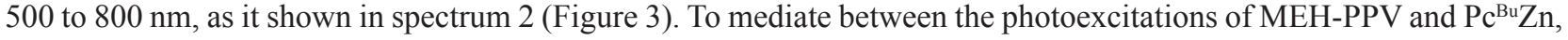
nearly resonant conditions are employed using green and red light-emitting diodes (LEDs) with the peak wavelengths close to the peak absorption of MEH-PPV (Figure 3, spectra 1 and 4) and $\mathrm{Pc}^{\mathrm{Bu}} \mathrm{Zn}$ (Figure 3, spectra 2 and 3).

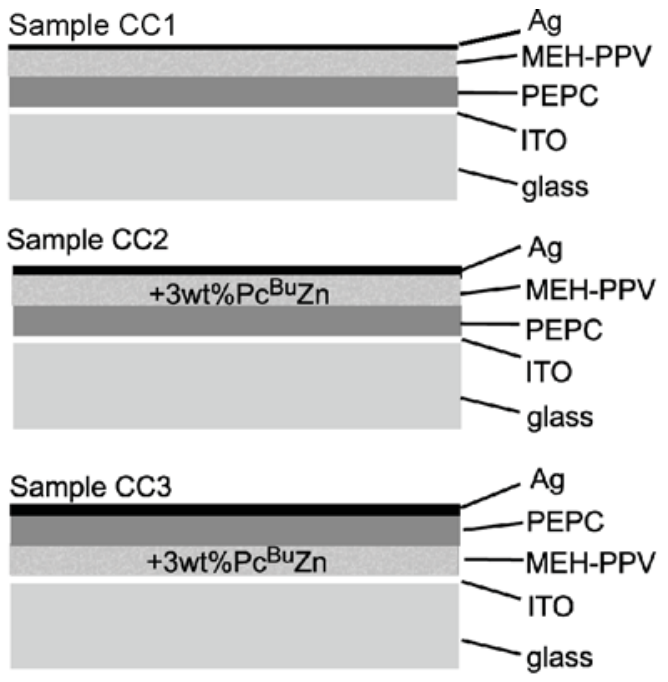

Figure 2. Schematic structure of the samples used in experiments.

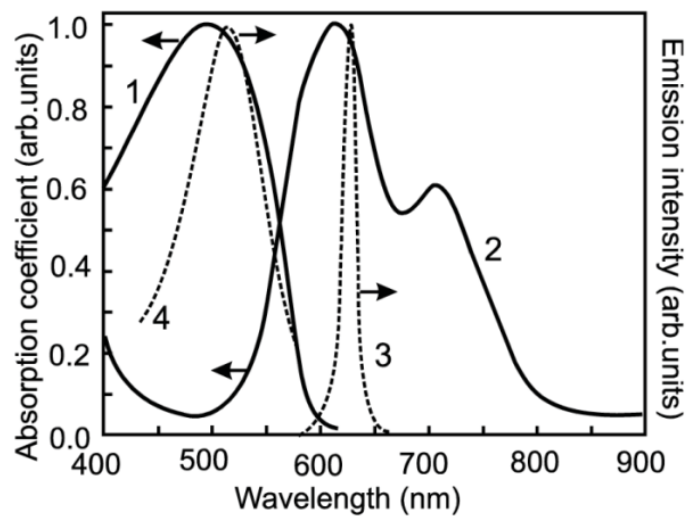

Figure 3. Absorption of MEH-PPV (1) and $\mathrm{Pc}^{\mathrm{Bu}} \mathrm{Zn}$

(2) compared with the red (3) and green (4) LED emission spectra.

The same green and red light is used for generation of photocurrent (PC) and PV signals which ones are directly registered at the ITO and Ag electrodes by an amplifier followed by a digital oscilloscope. With the electrical circuitry used, the positive sign of PV indicates that the Ag contact becomes positive with respect to ITO. The LEDs were controlled by the external bias in the form of square-function pulse. Light pulse lengths of $230 \mathrm{~s}$ and $300 \mathrm{~ms}$ to $100 \mathrm{~s}$ were used for the PC and PV measurements, respectively, with rise and fall times of 1ms. The PC and PV signals were supplied to a voltmeter, followed by a digital oscilloscope and stored on a computer. The electrical circuitry details are given elsewhere [13].

Several decay transients, taken with a single light pulse, were stored on a computer with the time interval of 20 and 40 min between the consecutive pulses for samples CC2 and CC3, respectively, which were greater than the PC and PV decay times. Then the stored transients were averaged as many times as required to achieve a given signal-to-noise ratio. 
The electrical loss tangent $(\tan \delta)$, inherent to dispersion in electrical conductivity, and capacitance $(C)$ of the sandwich structure were taken employing standard techniques at $1-\mathrm{kHz}$ rf voltage ranged from 1 to $30 \mathrm{~V}$, both in dark and under the LED illumination. The values of $\Delta \tan \delta=\left(\tan \delta_{P H}-\tan \delta_{0}\right) / \tan \delta_{0}$ and $\Delta C=\left(C_{\mathrm{PH}}-C_{0}\right) / C_{0}$ were analyzed, where the subscripts 0 and $\mathrm{PH}$ correspond to appropriate values measured before and at the illumination, respectively. All measurements were recorded at room temperature.

\section{Results and discussion}

The PC transients are shown in Figure 4. Samples CC1 have exhibited the lowest peak of PC signal, whereas samples CC2 have shown the highest one. The PC decays, measured after the light is turned off, are also markedly different, depending on the polarity of the applied voltage $U$. As seen in Figure 4, sample CC3 has exhibited the longest decay.

The peak PC $j_{\max }$ depends on the strength of the applied electric field (Figure 5), and the curves appear to be linear in the double-log coordinates, so that $j_{\max } \sim U^{m}$ with $m>2$.
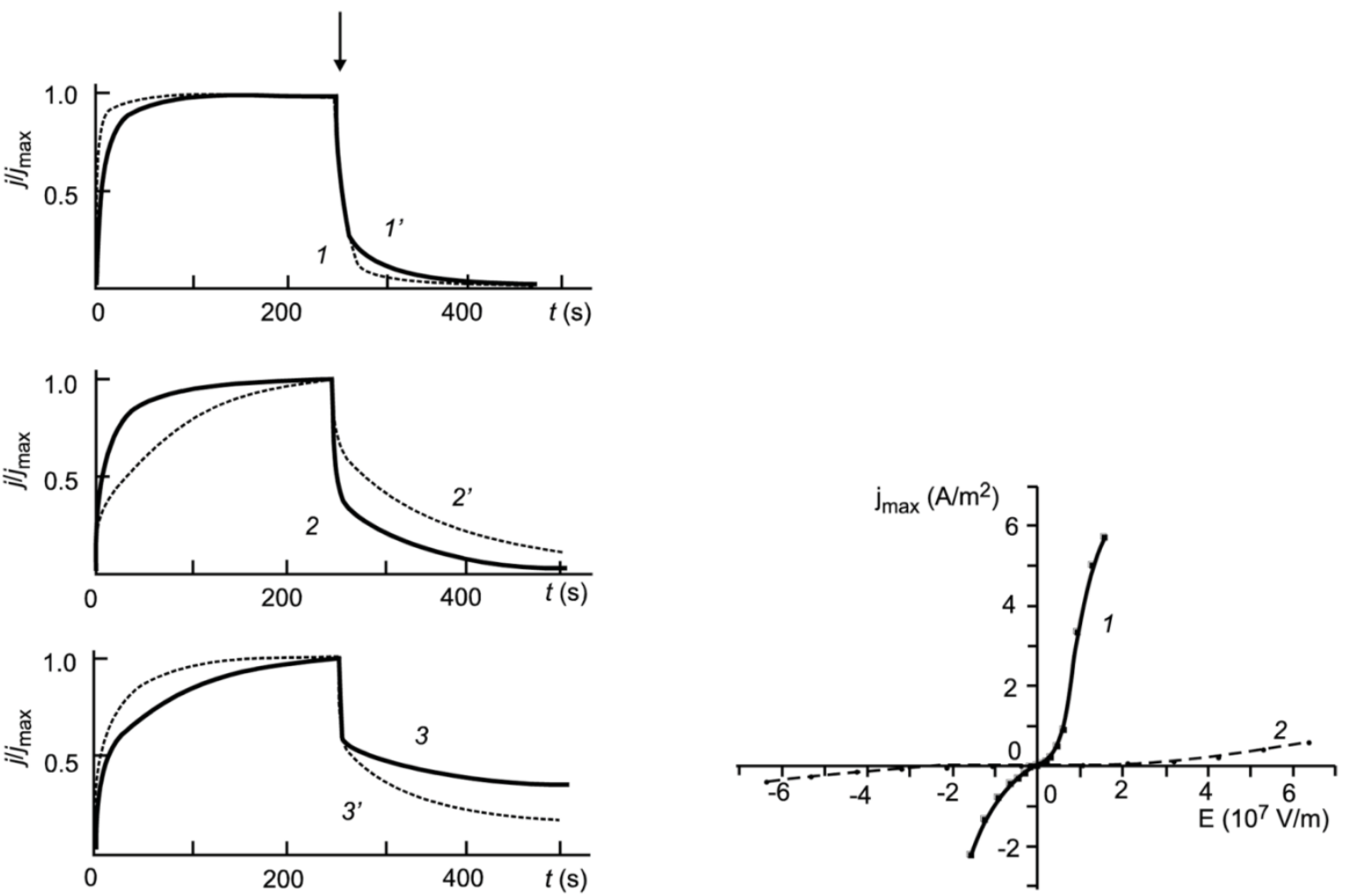

Figure 4. Normalized $P C$ transients taken in samples CC1 (1, 1'), CC2 $\left(2,2^{\prime}\right)$ and $\mathrm{CC} 3(3,3$ ') at the positive (1 to 3 ) and negative ( 1 ' to 3 ') polarity of applied voltage $U$ on the ITO electrode. The green LED with a peak intensity of $20 \mathrm{~W} / \mathrm{m}^{2}$ is used as illumination Figure 5. The peak PC value $j_{\max } v s$ applied electric field strength $E$ taken in samples CC2 (1) and CC3 (2) at illumination with the green LED having a peak intensity of $20 \mathrm{~W} / \mathrm{m}^{2}$. source. Arrow indicates the moment when the light is turned off.

The PV transients are shown in Figures 6-8. The most important, samples CC1, made-up without the phthalocyanine complex, exhibited a very weak PV with very short rise and decay times (Figure 6).

The PV signal is substantially greater in samples CC2 and CC3, the PV transients depend rather sensitively on the illumination wavelength (Figures 7 and 8). It may thus be concluded that doping of MEH-PPV with the Pc ${ }^{\mathrm{Bu}} \mathrm{Zn}$ complex enhances PV, even in the spectral range of a maximum absorption of MEH-PPV. Next, the PV transient shapes have changed considerably when the transients are taken repeatedly, although the decay relaxes to zero value in all cases. This may be considered to be a memory effect during consecutive illuminations.

It is also found that, after the LED light is switched on, the tan $\delta$ and $C$ values increase symbatically. These ones are completely relaxed after the light is switched off. Figure 9 illustrates the changes in tan $\delta$ upon illumination. It is seen that, similar to the PC and PV transients, samples CC1 posses the lowest times of the photo-dielectric response. According the data presented in Figures 4 and 9, it can concluded that, in contrast to samples CC1, samples CC2 and 
CC3 contain deep traps capturing photoexcited carriers and slowing down the transients. The traps are obviously due to $\mathrm{Pc}^{\mathrm{Bu}} \mathrm{Zn}$ complexes. This is also supported by the PV data shown in Figures 6-8, illustrating that the PV kinetics is symbate with the kinetics of $\tan \delta$ and $C$ taken after light is switched on and switched off. Therefore, $\mathrm{Pc}{ }^{\mathrm{Bu}} \mathrm{Zn}$ in $\mathrm{MEH}-\mathrm{PPV}$ has sensitizing properties, which are most likely to be due to deep traps arisen in the vicinity of the $\mathrm{Pc}^{\mathrm{Bu}} \mathrm{Zn}$ molecule.

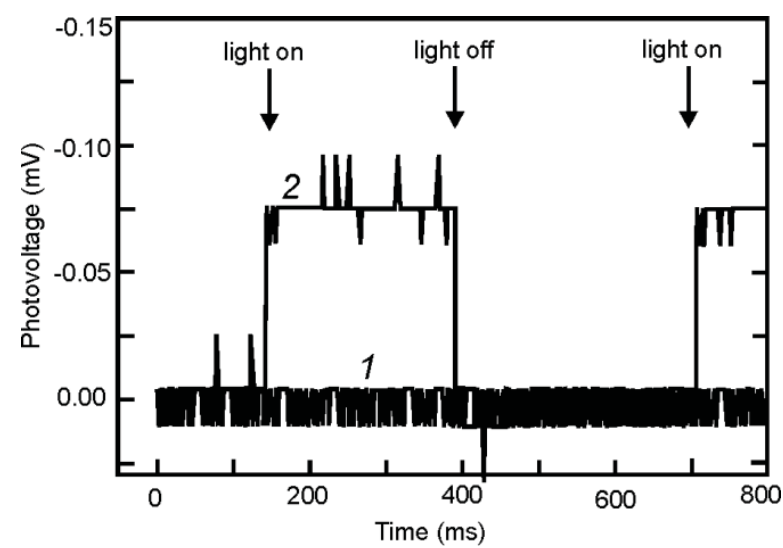

Figure 6. Time-dependent PV of sample CC1 at the red (1) and green (2) LED illumination. Curve 2 is arbitrarily shifted in the vertical direction.

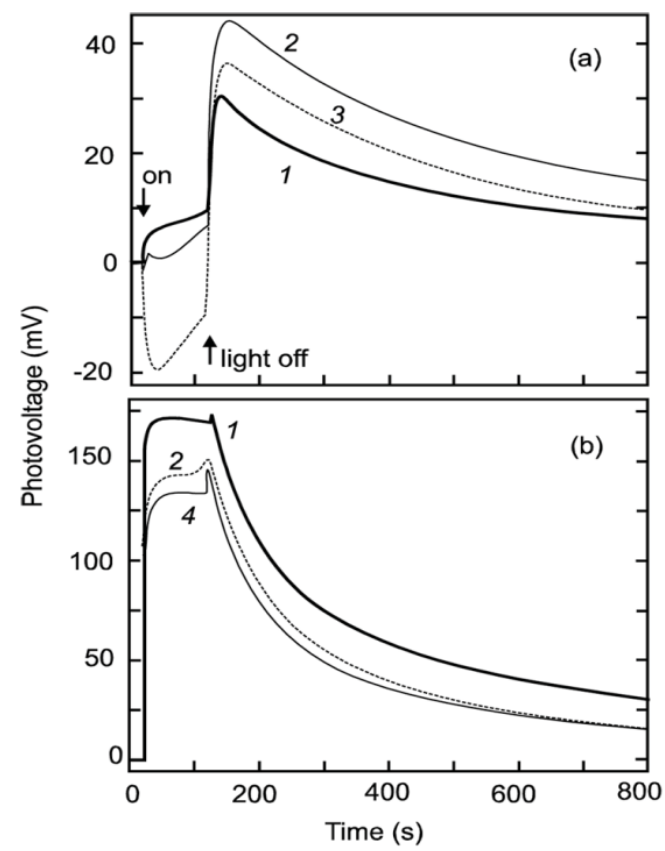

Figure 8. Time-dependent $\mathrm{PV}$ of sample $\mathrm{CC} 3$ at the red (a) and green (b) LED illumination. Curve numbers indicate the consecutive decay traces captured with the time intervals of 40 minutes.

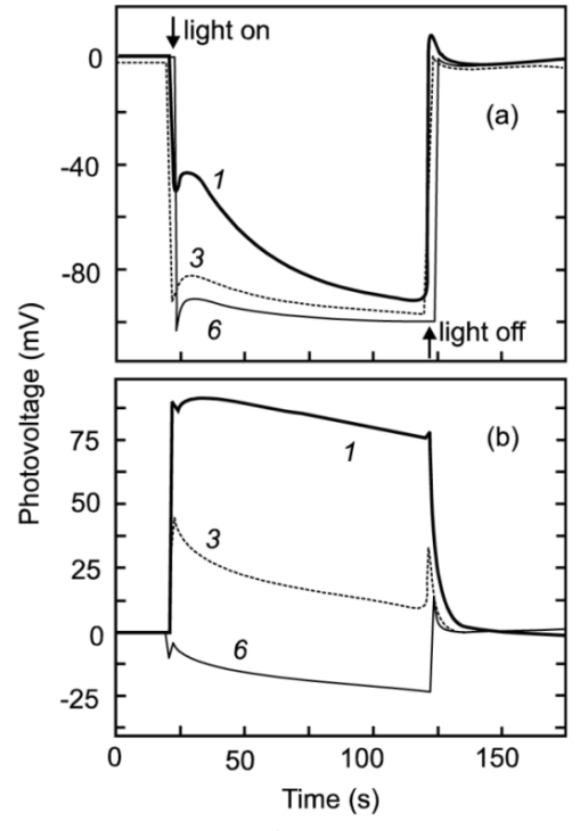

Figure 7. Time-dependent PV of sample CC2 at the red (a) and green (b) LED illumination. Curve numbers indicate the consecutive decay traces captured with the time intervals of $20 \mathrm{~min}$.

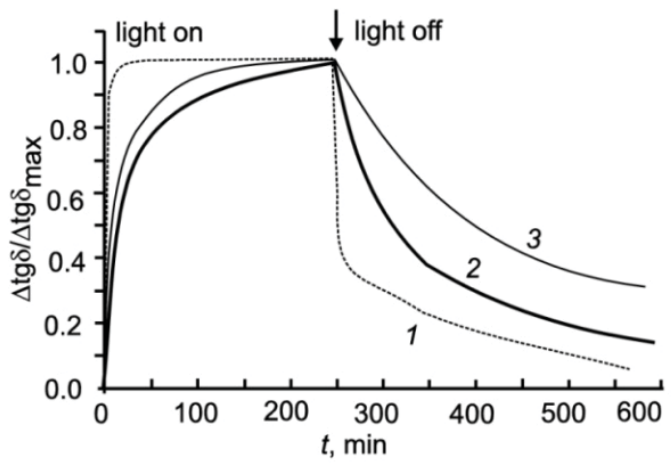

Figure 9. Time-dependent $\Delta \tan \delta$ of samples CC1 (1), CC2 (2) и CC3 (3) at the green LED illumination. 


\section{Conclusions}

$\mathrm{Pc}^{\mathrm{Bu}} \mathrm{Zn}$-sensitized MEH-PPV heterostructures have a superior photovoltaic performance in comparison with MEH-PPV structures. It is suggested that zinc octabutylphthalocyanine complexes incorporated into MEH-PPV produce deep traps localized at the $\mathrm{Pc}^{\mathrm{Bu}} \mathrm{Zn}$ molecule. The trap-mediated photo-electric and photo-dielectric transients exhibit prolonged transient times, indicative of trap-induced capture of charges. This charge turns out to screen the electric field developed at the hetero-interface and the barrier field due to metal contacts. The latter fact is capable of reversing the photovoltage sign due to prolonged light illumination.

\section{References}

1. Huang, C.; Wang, N.; Li, Y.; Li, C.; Li, J.; Liu, H.; Zhu, D. A New class of conjugated polymers having porphyrin, poly(p-phenylenevinylene), and fullerene units for efficient electron transfer. Macromolecules, 2006, 39, pp. 5319-5325.

2. Braun, D.; Heeger, A.J. Visible light emission from semiconducting polymer diodes. Applied Physics Letters, 1991, 58, pp. 1982-1984.

3. Yu, G.; Zhang, C.; Heeger, A.J. Dual-function semiconducting polymer devices: Light-emitting and photodetecting diodes. Applied Physics Letters, 1994, 64, pp. 1540-1543.

4. Sariciftci, N.S.; Braun, D.; Zhang, C.; Srdanov, V.I.; Heeger, A.J.; Stucky, G.; Wudl, F. Semiconducting polymerbuckminsterfullerene heterojunctions: Diodes, photodiodes, and photovoltaic cells. Applied Physics Letters, 1993, 62, pp. 585-588.

5. Shi, Q.; Hou, Y.; Li, Y.; Feng, Z.; Liu, X. Wavelength dependence of transient photovoltage polarity. Physics Letters A, 2008, 372, pp. 5853-5856.

6. Iyengar, N.A.; Harrison, B.; Duran, R.S.; Schanze, K.S.; Reynolds, J.R. Morphology evolution in nanoscale lightemitting domains in MEH-PPV/PMMA blends. Macromolecules, 2003, 36, pp. 8978-8985.

7. Maltsev, E.I.; Brusentseva, M.A.; Rumiantseva, V.D.; Lysenko, D.A.; Berendiayev, V.I.; Mironov, A.F.; Novikov, S.V.; Vannikov, A.V. Electric phosphorescence if aromatic polyimides doped with Pt-porphyrins. High-molecular compounds, 2006, 48, pp. 254-259 (in Russian).

8. Marletta, A.; Goncalves, V.; D’ebora, T. Photoluminescence of MEH-PPV/PS Blends. Brazilian Journal of Physics, 2004, 34, pp. 697-698.

9. Kumar, A.; Bhatnagar, P.K.; Mathur, P.C.; Tada, K.; Onoda, M. Improved electrical and optical properties of MEH-PPV light emitting diodes using Ba buffer layer and porphyrin. Applied Surface Science, 2006, 252, pp. 3953-3958.

10. Kazukauskas, V. Investigation of carrier transport and trapping by oxygen-related defects in MEH-PPV diodes. Semiconductor Science and Technology, 2004, 19, pp. 1373-1377.

11. Davidenko, N.A.; Spitsina, N.G.; Lobach, A.S.; Breusova, M.O.; Tomilova, L.G.; Yakushchenko I.K. Electrical conductivity and photoconductivity of films of composites based on poly[2-methoxy-5-(2'-ethylhexyloxy)1,4-phenylenevinylene] with additions of zinc octabutyl phthalocyanine and $\mathrm{C}_{60^{\circ}}$. Theoretical and Experimental Chemistry, 2006, 42, pp. 277-281.

12. Grazulevicius, J.V.; Strohriegl, P.; Pielichowski, J.; Pielichowski, K. Carbazole-containing polymers: Synthesis, properties and applications. Progress in Polymer Science, 2003, 28, pp. 1297-1301.

13. Podolian, A.; Kozachenko, V.; Nadtochiy, A.; Borovoy, N.; Korotchenkov, O. Photovoltage transients at fullerenemetal interfaces. Journal of Applied Physics, 2010, 107, pp. 093706-093713. 